\title{
Evolution of Large and Small Scale Magnetic Fields in the Sun
}

\author{
Peter A. Fox, Michael L. Theobald and Sabatino Sofia \\ Center for Solar and Space Research, Yale University, P.O. Box 6666, \\ New Haven, CT 06511 USA
}

\begin{abstract}
This paper will discuss issues relating to the detailed numerical simulation of solar magnetic fields, those on the small scale which are directly observable on the surface, and those on larger scales whose properties must be deduced indirectly from phenomena such as the sunspot cycle. Results of simulations using the ADISM technique will be presented to demonstrate the importance of the treatment of Alfvén waves, the boundary conditions, and the statistical evolution of small scale convection with magnetic fields. To study the large scale fields and their time dependence, the magnetic resistivity plays an important role; its use will be discussed in the paper.
\end{abstract}

\section{Introduction}

In attempting to understand the magnetic activity on stars, it is inevitable that we begin by searching for an understanding of the Sun. In turn, the Sun presents us with a very complex array of features related to magnetic activity. From a theoretical viewpoint, these features separate naturally into a large scale component and a small scale component. Both components are important in their own right, but the key to a realistic understanding of magnetic activity almost certainly lies in the interaction between the two scales.

The evolution of the large scale solar magnetic field divides into two timescales: that of the solar cycle, and one of a much longer (and presently unknown) timescale. Current theoretical models of the large scale field are necessarily simplified, often drastically, in order to make reasonable progress. On the other hand the small scale field, which can be constrained by observations, can be modeled in some detail. In particular, we can do both qualitative and quantitative studies in an attempt to understand how the magnetic field and the surrounding flow interact.

An understanding of both scales, even perhaps an incomplete one, will be important in analyzing the components of the solar dynamo process. Current dynamic 
models do not give a satisfactory explanation of even the simplest solar cycle features for a number of reasons (see Chan, these Proceedings). Unfortunately, since the kinematic models lack predictive capabilities, further investigation is required. This paper will discuss some limited aspects of the large scale field but will mainly discuss the small scale fields.

\section{Large scale magnetic fields}

Because of the tremendous uncertainty in the interior structure of the solar magnetic field, much closer attention should be given to calculating the evolutionary aspects of those fields. Since the understanding of angular momentum transport in stars is increasing rapidly (Endal and Sofia, 1976; Pinsonneault et al., 1989), the possibility of constraining the magnetic component becomes a reasonable next step. There is strong evidence (observational and implied theoretical) for the existence of internal differential rotation and large scale magnetic fields in solar-type stars.

The implications of certain magnetic field structures, for example at the base of the solar convection zone, are crucial to popular scenarios for the solar dynamo. In addition, it is important to understand what magnetic flux (i.e. in the mean sense) the solar convection zone may have had during its evolution which can consequently be compared to solar-type stars.

One constraint on interior magnetic fields that is yet to be fully developed is helioseismology. Except for very high field strengths (of order MGauss) the direct impact on oscillation mode frequencies is small. However changes in the solar structure (which may be position dependent) due to magnetic fields are likely to produce measurable changes in the oscillation frequencies. In addition there are, as yet still only suggestive, variations of oscillation frequencies (at particular wavelengths) over the solar cycle (Libbrecht and Woodard, 1990).

\section{Small scale magnetic fields}

Studies of the small scale interaction of convection and magnetic fields require the solution of the fully compressible form of the Navier-Stokes equations coupled with Maxwell's equations (see, for example, Priest, 1982). We will not attempt to review the present state of knowledge in this area but suffice to say that rapid progress has been made in the last few years.

The numerical solution of the governing equations can be thought of as a two stage procedure, one of a qualitative nature using calculations that have two spatial dimensions (with or without axisymmetry), and the other of a quantitative nature that includes all three dimensions. Both approaches are useful but we will make a few comments about the former in this paper. In particular, we know from existing work that many magnetic field related features are evident in two 
dimensional calculations (compared to three dimensions) even though some details are wrong (e.g. reconnection).

It is important to have a good qualitative understanding of the physical conditions, as well as of the limitations of the numerical and physical approximations, before progressing to more complex models. Part of this procedure should lead to better approximations or scaling relationships when investigating larger scale fields (and flows).

The results presented here are from $2 \mathrm{D}$ simulations in cartesian geometry using the ADISM (Chan and Wolff, 1982) method. More details on these and related calculations can be found in Fox et al. (1990). The usual features such as expulsion of magnetic flux, concentration of flux which is stronger in downflows and weaker, but present, in upflows (depends on the magnetic resistivity, $\eta$ which we will emphasize shortly), and magnetic reconnection are all evident in the simulations. In addition, beyond a threshold magnetic flux (here measured as $5 \%$ of the surface gas pressure), there is a time dependence manifested as a continuous transformation of weak (but not necessarily uniformly distributed) fields into small regions of intense fields (which implies the ubiquity of fibril-type fields) which in turn decay, etc.

These small scale interactions show "complex" exchange between kinetic and magnetic energies which, when analyzed, should give direct information on wave interaction and perhaps indirect information on dynamos if the features actually can be extended to larger scales.

One quantity which is very uncertain in simulations of magnetohydrodynamics is the magnetic resistivity. It limits the concentration of magnetic flux, smooths the fibril field, influences the decay of large scale fields, and most importantly limits the time steps in numerical schemes, usually to satisfy some numerical stability criterion. Because of the important role that the resistivity plays, we would like to encourage detailed investigations to determine the answers to a number of questions: Is there turbulent enhancement of resistivity? What is its magnitude and form? Is the Alfvén hypothesis correct, i.e. is the dissipation of magnetic and kinetic energy the same on small scales?

Figure 1 shows the effect of changing the magnetic resistivity in a 2-D simulation. The vector potential, which in this case gives the lines of magnetic force, is overlayed with the velocity vector field. There are obvious differences between the field structures that develop.

As the strength of the magnetic field is increased there is strong evidence of overstable oscillations and a preference for convection cells of small aspect ratio (width to depth) to develop. This is not a new result but there is a subtle dependence on other quantities, such as magnetic resistivity and boundary conditions, that also needs further investigation (see Fox et al., 1990).

Another feature of these simulations is the presence of waves, i.e Alfvén, sound and MAG. Figure 2 shows the magnetic energy spectra for two simulations. The low frequency peaks are magnetic and the high frequency are from sound wave exchange. 
One important result for this type of modeling is that the initial distribution of magnetic field does not determine the final configuration once the simulation has been allowed to "relax" (see Chan and Sofia, 1986). The main external constraint on the magnetic field arises from the boundary conditions. Unfortunately this is a very difficult problem to treat accurately and we are left with compatibility relations that are only partially realistic, for example, imposed radial field component at the surface (but non-zero horizontal component).

The statistical relaxation time of simulations of compressible convection depends on the mass within a layer and the number of pressure scale heights. So far no attempts have been made to determine the influence of magnetic fields on this process, except that for small magnetic fields there should be no difference. Preliminary indications are that the relaxation time can be shortened by strong magnetic fields, essentially because of a combination of the Poynting flux carrying a fraction of the flux, and the smaller aspect ratio cells mentioned previously allow more rapid communication between the lower and upper layers. Unfortunately we have not yet fully investigated the influence of a large number of pressure scale heights, and so some of our conclusions are preliminary.

\section{Connecting the two scales}

There are a number of important physical features that occur in large scale magnetic fields, or are implied by our primitive understanding of the solar dynamo. One of these is to determine the region of influence of the solar dynamo, i.e. how important is it to know about the sub-convection zone field? What interaction occurs at the boundary and do we have to worry about generating a cyclic field there? In addition, can we evaluate the concept of the $\alpha$-effect, either on small scales or large scales using detailed models?

\section{Summary}

In this paper we have attempted to promote the use of numerical simulations as a tool, not only for modeling surface features, but for determining more global properties associated with the interaction of convection and magnetic fields. Since there is less adjustability (in terms of parameters) in current numerical models, compared to analytical or simplified numerical approximations, we should be able to identify structures and effects that can be modeled on the larger scales and also determine which features are unimportant and neglect them. In essence we view this approach as the development of a numerical "mean field" theory.

We consider the statistical evolution of magnetic fields, the influence of boundary conditions, and the role of magnetic resistivity in determining magnetic field structures to be crucial to the understanding of the solar dynamo. Finally, it is essential that we develop ideas about the importance of "waves" (sound, Alfvén, MAG, etc.) as a contributor to both large scale fields and the solar dynamo. These 

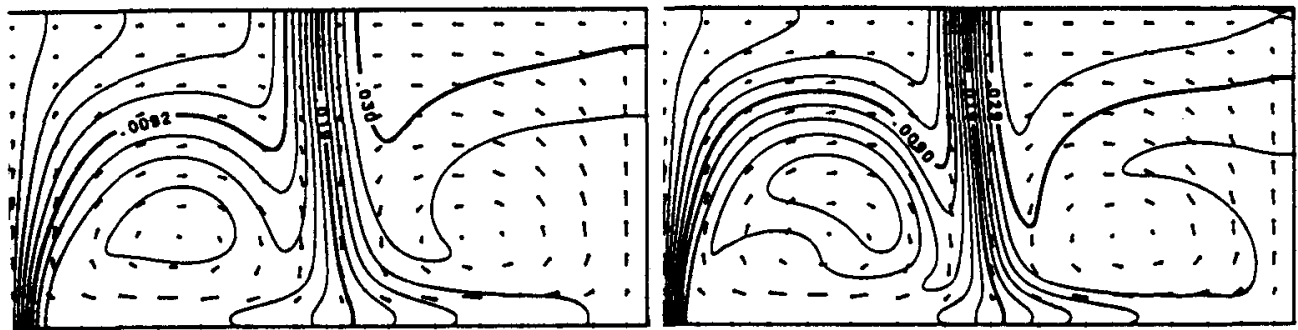

Fig. 1. Comparison of two simulations of two dimensional compressible convection interacting with magnetic fields. Using the same initial magnetic flux but different magnetic resistivities (factor of two greater in the left panel). Only a subset of the domain is shown.
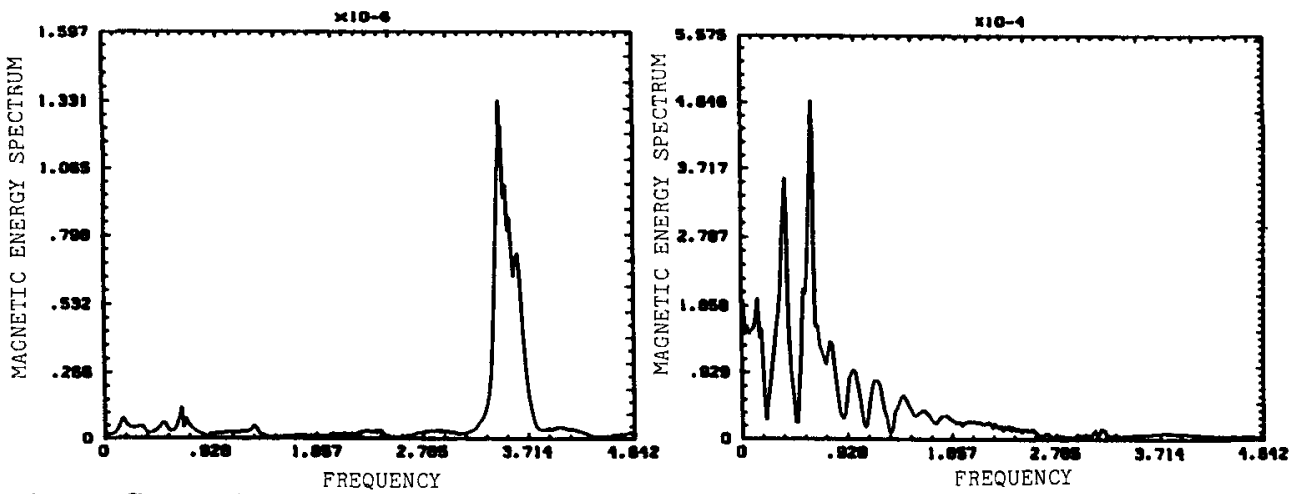

Fig. 2. Comparison of two magnetic energy spectra from simulations similar to Fig.1. The left panel is for weak initial field and the right is for strong field, using the same magnetic magnetic resistivities.

waves presently constitute a significant barrier to numerical simulations on solar cycle timescales and so a "non-wave" approximation would be extremely useful. This work is supported by grants from NASA (NAGW-777) and the USAF (AFOSR-88-0054).

\section{References}

Chan, K. L. , Sofia, S.: 1986, Astrophys. J. 307, 222

Chan, K. L. , Wolff, C. L.: 1982, J. Comp. Phys. 47, 109

Endal, A. S. , Sofia, S.: 1976, Astrophys. J. 210, 184

Fox, P. A., Theobald, M. L. , Sofia, S.: 1990, submitted to Astrophys. J.

Libbrecht, K. G., Woodard, M. F.: 1990, Nature 345, 779

Pinsonneault, M. H. et al.: 1989, Astrophys. J. 338, 424

Priest, E. R.: 1982, Solar Magnetohydrodynamics, (Reidel, Dordrecht) 